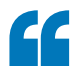

Mutation analysis should be the standard of care for all paediatric patients with SRNS

NEPHROTIC SYNDROME

\title{
Novel monogenic causes of Galloway-Mowat syndrome
}

Researchers have identified causative mutations in the four KEOPS subunit genes - OSGEP, TP53RK, $T P R K B$ and $L A G E 3$ - in patients with Galloway-Mowat syndrome (GAMOS). This syndrome is characterized by early-onset steroid-resistant nephrotic syndrome (SRNS) in combination with microcephaly and brain anomalies. The KEOPS complex catalyzes a universal post-transcriptional modification of transfer RNA and has roles in the regulation of gene transcription and genome maintenance.

To identify novel monogenic causes of SRNS, Friedhelm Hildebrandt and colleagues combined whole-exome sequencing with homozygosity mapping in 91 families. "This unbiased approach enables the unravelling of entirely novel and unexpected pathogenic pathways,' says Hildebrandt.

The researchers identified mutations in genes encoding KEOPS subunits in 37 patients from 32 different families. These patients had a highly concordant clinical phenotype of GAMOS, comprising early-onset SRNS, primary microcephaly, developmental delay, and additional dysmorphic or skeletal features. Renal biopsy samples from 19 of these patients showed focal segmental glomerulosclerosis (FSGS), diffuse mesangial sclerosis or other glomerular lesions as well as partial podocyte foot-process effacement. "The unusually high number of affected patients identified in our study suggests that a large percentage of patients displaying this specific combination of phenotypes have mutations in KEOPS-encoding genes," says Hildebrandt.
CRISPR-Cas9 knockout of orthologous KEOPS subunit genes in zebrafish and mice reproduced the primary microcephaly phenotype but not the renal phenotype seen in patients with GAMOS. The researchers suggest that early lethality in these models could have masked the renal involvement that might have been seen in older animals.

In human podocytes, knockdown of KEOPS subunit genes led to reduced proliferation and migration, impairment of protein biosynthesis, defects in the actin cytoskeleton, activation of the unfolded protein response, endoplasmic reticulum (ER) stress and increased apoptosis. "Our work identifies the first monogenic forms of SRNS that can be attributed to the ER stress pathway," comments Hildebrandt. "Other groups have shown that ER stress can be partially remedied by drug treatment. Therefore, our findings may point towards a potential route for future treatment of patients with SRNS and mutations in KEOPS-encoding genes.”

The researchers also observed activation of DNA damage response (DDR) signalling in podocytes with knockdown of KEOPS subunit genes. "This finding suggests that additional, non-canonical functions of the KEOPS complex exist, and that these contribute to the pathogenesis of SRNS," says Hildebrandt.

"Activation of the DDR pathway has been described in some monogenic forms of microcephaly, but it has not previously been implicated in the pathogenesis of FSGS and podocyte dysfunction. Our results show that this cellular defect may be partially mediated by an interaction between

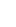

\section{.}

\section{(n)}
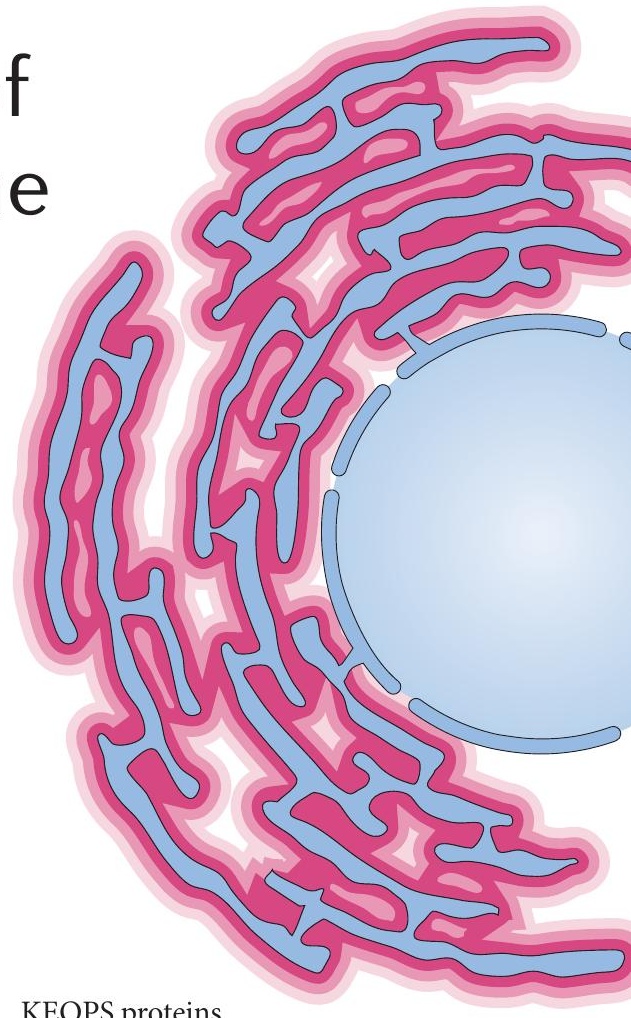

KEOPS proteins

and the DNA repair protein

PARP1."

The researchers conclude that mutations in KEOPS subunit genes are new monogenic causes of GAMOS. "Given the technical advances and significant cost reductions that have occurred over the last few years, we propose that mutation analysis should be the standard of care for all paediatric patients with SRNS," says Hildebrandt. "Identification of novel monogenic causes of SRNS will increase the number of patients in whom a causative single gene mutation can be identified and could be used to help guide therapy."

Ellen F. Carney

ORIGINAL ARTICLE Braun, D. A. et al. Mutations in KEOPS-complex genes cause nephrotic syndrome with primary microcephaly. Nat. Genet. http://dx.doi.org/10.1038/ng.3933 (2017) FURTHER READING Vivante, A. \& Hildebrandt, F. Exploring the genetic basis of early-onset chronic kidney disease. Nat. Rev. Nephrol. 12, 133-146 (2016) 